\title{
Not Cure but Heal: Music and Medicine
}

\author{
Paulo Estevao Andrade ${ }^{1}$, Joydeep Bhattacharya ${ }^{1, *}$ \\ ${ }^{1}$ Department of Psychology, Goldsmiths, University of London, London, UK \\ (*Contact: j.bhattacharya@gold.ac.uk)
}

"Do you know that our soul is composed of harmony?"

Leonardo Da Vinci,

Despite evidence for music-specific mechanisms at the level of pitch-pattern representations, the most fascinating aspect of music is its transmodality. Recent psychological and neuroscientific evidence suggest that music is unique in the coupling of perception, cognition, action and emotion. This potentially explains why music has been since time immemorial almost inextricably linked to healing processes and should continue to be.

\section{Introduction}

Music captures our attention almost automatically, moves us emotionally, activates a wide range of brain regions, both at cortical and subcortical level, and engages a spectrum of processes pertaining to attentional, perceptual, memory, emotional, sensorimotor, mental simulation, perception-action, and communication (Koelsch, 2012). Therefore, it is not surprising that music has therapeutic effects on the physiological and psychological well beings of individuals (Wheeler, 1996). In fact, music therapy is defined as a systematic process in which carefully controlled music is used "in the treatment, rehabilitation, education and training of children and adults suffering from physical, mental or emotional disorder” (Alvin, 1975, p.4; see also Bunt and Stige, 2014). The American Music Therapy Association defines it as "the clinical and evidence-based use of music interventions to accomplish individualized goals within a therapeutic relationship by a credentialed professional who has completed an approved music therapy program” (Juslin and Sloboda, 2011).

Despite anthropological and ethnomusicological evidence showing ancient belief on the healing powers of music (Gouk, 2000; Merriam, 1964), music therapy is a 
relatively new research field whose formalization of education and research started only a few decades ago (Bunt and Stige, 2014). Music can have widespread beneficial effects, but the underlying mechanisms are not well understood or even adequately investigated. Therefore, music therapy as such was not considered amongst the mainstream medical intervention techniques till recently. In fact, medical practitioners in Western and westernized societies, differently from the non-Western medical and healing traditions (Gouk, 2000), have been careful and even skeptical in considering music as an effective medium of healing and/or fitting into scientific procedures (Bonny, 1986). The reason for this might reside on the fact that the final product of music is the "invisible" (sounds) with a great power of evoking emotions, making music the art that best lends itself to abstraction of our feelings. Indeed, for all pre-literate cultures, music had a sacred character, conveying cosmogonic and existentialist meanings, serving as an articulation point between the physical and the metaphysical (Andrade, 2004). Although psychophysiological effects of music (e.g., on electrocardiogram and blood pressure) were documented almost a century ago (Hyde and Scalapino, 1918), it is only from 1990s that an increasing amount of empirical studies investigating music-based intervention methods has taken place (Thaut, 2005). Investigations on music-therapy, however, have faced two major problems (Hillecke et al., 2005). The first is related to specificity, or a lack of it, i.e. the question whether observed outcomes are due to music-specific ingredients or to other factors common in the treatment of psychological disorders. In other words, the problem of specificity is a result of the adoption of the psychotherapy research tradition which did not control for unspecific factors influencing music-based intervention outcomes, such as extra-therapeutic aspects, therapeutic relationship, expectancy and placebo effects (Hillecke et al., 2005). The second problem is due to the theoretical heterogeneity among several music therapy approaches such as psychoanalytic music therapy, humanistic music therapy, behavioral music therapy, Nordoff-Robins music therapy, and music medicine. Such heterogeneity creates difficulties not only for an effective communication between music therapy centers but also in the search for working ingredients underlying successful music-based interventions, a crucial knowledge that could help both improving current interventions and guiding new hypotheses. Accordingly, the best way to handle these problems is to develop theories which are both coherent with available empirical knowledge and amenable to be tested and falsified. 
Cognitive neuroscience has emerged as a promising scientific field which could give to music therapy its deserved scientific status. Particularly from 2000s, the development of advanced neuroimaging techniques has yielded important insights into the neural correlates of both listening to and engaging with music. It has been shown that music involves a multitude of brain areas dedicated to perception, cognition, i.e. short and long-term memory mechanisms, language, visuospatial processing, sequential processing and prediction, etc., motor skills, sensory-motor integration (Andrade and Bhattacharya, 2014; Levitin and Tirovolas, 2009; Zatorre, 2005). Studies on the processing of musical emotions reveal activations of ancient structures in the limbic (e.g. hippocampus, amygdala) and paralimbic (e.g. caudal orbitofrontal cortex, insula, temporal pole, parahippocampal gyrus) brain regions (Koelsch, 2010) as well as associated neurochemical changes related to reward, motivation, pleasure, stress and arousal (e.g. fear) (Chanda and Levitin, 2013).

These findings from cognitive neuroscience of music have served as the basis for the development of music-based interventions to ameliorate memory, attention, language, spatial awareness, motor and executive functions (Koelsch, 2009; Shannon, 2010; Thaut, 2005). Psychophysiological effects of music-evoked emotions are guiding interventions aimed at reducing anxiety, stress, pain, treatment of depression, etc. (Chanda and Levitin, 2013; Koelsch, 2015).

A neurological music therapy has been proposed and defined as "the therapeutic application of music to cognitive, sensory, and motor dysfunctions due to neurologic disease of the human nervous system” (Thaut, 2005, p.126). In the same line, other authors offer explanative models of the factors or working ingredients of music-therapy underlying the positive effect of music on the psychological and physiological health of individuals, such as modulation of attention, emotion, cognition, behavior, and communication (Hillecke et al. 2005; Koelsch, 2009).

There has been an upsurge in the number of research publications on the main neurobiological and neurocognitive principles underlying evidence-based music therapy. Nonetheless, it is important to keep in mind that music therapy is a growing and multidisciplinary field that builds on knowledge from acoustics to neurobiology and biomedical research, from psychology to neurology, from sociology to musicology and ethnomusicology (Hillecke et al., 2005). In this chapter we intend to offer an integrated and convergent framework in which we directly address the connections of universal features and functions of musical behavior with the neuroscientific perspective of music 
therapy, linking anthropological/ethnomusicological and developmental data with the set of already known working ingredients underlying successful music therapy. Our main aim is to provide readers with the relevant references and sufficient information to enable them to form a theoretical and empirical framework with which the available research findings could be critically evaluated and testable hypotheses be formulated for future research.

\section{Musical behavior: universal, ancient and precocious}

The notion that music is a universal behavior which goes back to the origins of human species appears undisputable (Conard, Malina, and Münzel, 2009; Mithen, 2006). Musical ubiquity across space and time has led many scholars, since Darwin's publication of Descent of Man in 1871, to propose that music might be a biological adaptation (Mithen, 2006; Wallin et al., 2001; but see also Justus and Hutsler, 2005; Patel, 2010; Pinker, 1997). It is though debatable which musical traits were under selection pressure (Fitch, 2005; Honing et al. 2015; McDermott, 2008; Merker et al. 2015; Trainor, 2015).

One perhaps wonders why a book chapter on the therapeutic use of music would explore some universals and evolutionary issues of music. Strange as it may seem, this link is of crucial relevance for understanding the profound impact of music on human beings and, hence, its relevance and potential use in clinical and therapeutic settings. Our idea on the importance of understanding typicality of musical behaviors for the cognitive neuroscience of music is well illustrated in the epigraph by Shepherd (1994, p.9) "nothing in neurobiology makes sense except in the light of behavior”. In other words, naturally occurring, ancient and universal behaviors, as is the case of music and language, are presumed to be mediated by neural circuits with a deep evolutionary history. Thus, deepening the understanding of what is relevant in terms of behaviors feeds the knowledge of what is relevant in terms of neural circuits and vice-versa.

Actually, several features of musical behaviors that are consistent with an evolutionary account of music represent, in our view, the substrate from which the most cogent arguments for its therapeutic use arises. Music is universal across extant and extinct human cultures. Despite cultural idiosyncrasies, there are important similarities (universalities) in pitch and rhythm structures and in the functions of music across cultures, and most importantly, perception of and attraction for music emerge very early in ontogenetic development. Processing of musical patterns by infants is similar to that 
of adults so that they respond better to melodies in diatonic scales as well as to consonant patterns and to complex metric rhythm, and further they possess absolute pitch early in life which changes to relative pitch later and have long-term musical memory as well. Finally, there is evidence that structural components such as pitch contour and pitch interval are encoded automatically, even by non-musicians. Taken together these evidence suggests that our auditory pathways are likely to be hard-wired to deal with music-related stimuli (Andrade and Bhattacharya, 2003; Justus and Hutsler, 2005; McDermott and Hauser, 2005; Zatorre, 2005).

Music is also a nonverbal form of communication. Historically it is a shared group activity, spontaneous and improvisational involving sound-movement synchronizations (Juslin and Sloboda, 2011). Although music is usually defined in terms of an aural phenomenon based on patterned sound along pitch and time dimensions, there is, nevertheless, an emergent consensus is that music is inseparable from movement, i.e. it is both sonic and embodied (Cross, 2001; Dissanayake, 2009, Merker et al. 2015). Moreover, the deep links between music, emotion and movement occurring from infancy to adulthood are also universal.

These profoundly organic features of music together along with its polysemic nature are perhaps the principal reason why serving as an important medium of healing is amongst the universal functions of music across societies, including courtship, praying, mourning and instructing (Blacking, 1973; Cross, 2001; Gouk, 2000; Dissanayke, 2009).

\section{Music-and-movement therapy}

A main feature of music across cultures is that it is a shared activity involving body movements, such as toe-tapping, head-nodding, hand-clapping (even in solitary appreciation recently allowed by the phonographic industry) and mainly dance (Blacking, 1973; Cross, 2001; Mithen et al., 2006). In other words, when one moves, plays or sing along with music, the sensory experience of musical patterns is intimately coupled with action. Unsurprisingly some cultures employ terms to define music that are far more inclusive than the Western notion of music, like the word nkwa that for the Igbo people of Nigeria denotes "singing, playing instruments and dancing” (Cross, 2001, p. 29). Or take sangeet, the Sanskrit word for music, which literally means singing together. 
Moreover, developmental precursors of music in infancy through early childhood musical behaviors reveal an intense interest in music in the form of universal proto-musical behaviors which are exploratory and kinesthetically embedded and closely bound to vocal play and whole body movement. This scenario displaying an inextricable link between music, emotion and movement actually continues to unfold through adulthood by developing to some new forms of shared (or not) activities such as singing and dance (Dissanayake, 2000; Trehub, 2003; Trevarthen, 2000).

Indeed, in a way consistent with the view that music and dance are intertwined, some researchers propose that entrainment, i.e. coordination between internal and external rhythms (Merker et al. 2015), also referred to as sensorimotor synchronization (Repp and Su, 2013), constitutes the most distinctive musical behavior. It is further argued that entrainment is at the heart of protomusical behaviors and music evolution, being vital to organism's adaptation for conferring survival benefits originated from primary selection pressures, such as better perceptual and predictive capacities, and from secondary selection pressures such as facilitation of social interactions both at the level of mother-baby connections and group cohesion (Merker et al., 2015). Furthermore, similar to primary reward, like food and sex, with high adaptation value, music engages mesolimbic reward network (Koelsch, 2015; Zatorre and Salimpoor, 2013).

Consistent with the notion that music is both sound and action, and spontaneously elicits movements in the listeners, neuroimaging studies have frequently reported activations of motor areas in the brain, even during simple listening to music (Koelsch, 2009). Particularly bilateral frontal and inferior frontal activations, mainly premotor frontal areas BA6, dorsolateral prefrontal areas (BA8/9), as well as inferior frontal areas as Broca (BA44/45), insula, and more anterior middle and inferior frontal cortices (46/47), are frequently observed in non-musicians (Platel et al., 1997; Zatorre et al., 1994) and musicians (Zatorre et al., 1998) even during passive listening to pitch sequences or Bach's music (Ohnishi et al., 2001), and during a task of musical imagery (Halpern and Zatorre, 1999; Meister et al., 2004). Premotor cortex and supplementary motor areas and cerebellum are also activated during both reproduction (Sakai et al., 1999) and passive listening to rhythms (Chen et al. 2008).

More recent neurological findings have provided additional support for the existence of a natural link between sound and movement, with different sub-regions in the premotor cortex mediating distinct auditory-motor transformations. The ventral part 
of premotor cortex is particularly involved in anticipation and tap along with rhythms whereas the dorsal part is also recruited during movement synchronization and metrical organization. Even those sounds not clearly connected with a given action elicited activations in premotor sub-regions falling in between ventral and dorsal parts, supplementary motor areas and cerebellum as well (Chen et al. 2008).

Taken together, anthropological/ethnomusicological, developmental and neurological findings are consistent with the hypothesis that the capacity of music to spontaneously and unconsciously modulate motoric behavior is a relevant working ingredient of music therapy. This has been confirmed by the therapeutic potential of music in gait rehabilitation of stroke patients and other motoric problems such as those found in Parkinsons disease, autism, etc. For example, Thaut and colleagues (1997) found that combination of conventional physical therapy and rhythmic auditory stimulation where patients listen to a metronome or music tapes played over headsets while training their walking, has significantly ameliorated the walking patterns measured at post-test were compared to physical therapy alone.

According to Jankovic (2008) the four cardinal features of Parkinson's are: tremor at rest, rigidity, bradykinesia (the most important clinical feature characterized by slowness of movement) and postural instability, referred together as TRAP. The neurobiochemical signature of Parkinson's patients is a marked deficit in dopamine concentrations in the striatum (part of the subcortical structure in the brain known as basal ganglia).

There is evidence that the auditory-motor integration also occurs at the level of basal ganglia, i.e. amygdala, striatum (dorsal: caudate nucleus and putamen, ventral: nucleus accumbens) and globus pallidus via auditory association areas’ projections to these sub-cortical structures. The basal ganglia are particularly involved in the voluntary control of complex movements (Pinel, 2011), such as auditory sequencing and timing, musical rhythms (Janata and Grafton, 2003;Thaut, 2010; Zatorre et al., 2007) and speech and language as well (Enard, 2011). As pointed out by Thaut (2010) these pathways may play a critical role in the facilitative effect of music and auditory rhythm on motor output in Parkinson's disease.

Further, the basal ganglia is also a part of a timing network particularly involved in the extraction of durations using regular beats as a reference (e.g. perception of metrical rhythms), whereas the cerebellum is involved in the perception of absolute duration regardless of the presence of regular beats (Teki et al., 2011). Teki and 
colleagues (2011) have shown that a striato-thalamo-cortical timing network, in which striatum and supplementary motor areas (also known for its involvement in timing) are interconnected, is crucial for the beat-based duration perception. Of particular relevance here is the close neurobiological link between rhythmic abilities and Parkinson's disease which is characterized by a primary loss of dopaminergic neurones in a structure of the mesencephalon named substantia nigra (Hughes et al., 1992). More specifically, the nigrostriatal neurons, i.e. neurons from the dopaminergic pathway connecting substantia nigra to the dorsal striatum also known as mesostriatal dopamine pathway, are critical for interval timing so that Parkinson's patients are also impaired on time and rhythmic discrimination tasks (Teki et al., 2011). Actually, these connections between rhythmicity, motoric functions and dopamine have been confirmed by empirical findings that faster rhythmic auditory stimulation significantly enhanced gait velocity, cadence and walking pattern in Parkinson patients with and without medication (Thaut, 2010).

Since release of dopamine in the striatum is necessary for basal ganglia mediated motor behaviors, the evidence suggests that positive effects of music on impaired motoric abilities of Parkinson's patients include effects of rhythmicity on the mesostriatal dopamine pathway traditionally known for its motoric functions (planned movements) (Chanda and Levitin, 2013; Wise, 2009). There is an emerging agreement that the mesostriatal pathway also contributes to reward (Wise, 2009). So it is likely that mesostriatal pathway also contributes to motivation beyond motoric functions.

In this motoric context, let us briefly discuss another neurological disorder, Autism Spectrum Disorders (ASD). ASD is mainly characterized by the social communication impairments (difficulties in acquire language and its idiosyncratic use, and impaired social interaction) but often accompanied by stereotyped repetitive behaviors, and increasing evidence indicates that perceptual-motor impairments may be common in this disorder (Srinivasan and Bhat, 2013). For example, in the motor domain, patients suffering from ASD have problems with dual and multi-limb coordination, postural control, gait, and imitation and praxis (Srinivasan and Bhat, 2013). As perception-action is intricately coupled for music, potentially it could be utilized to facilitate motoric processes in autistic patients (Overy et al., 2009; Srinivasan, and Bhat, 2013).

\section{Music-and-emotion therapy}


Possibly the most fascinating aspect of music is its power to induce emotions and influence mood. Music can leave people happy or sad, calm or anxious. Music’s extraordinary ability to evoke powerful emotions is likely the main reason why we listen to music and why it is generally referred to as the "language of emotions" (Juslin and Sloboda, 2011).

For example, Panksepp (1995) asked hundreds of young men and women why they felt music to be important in their lives, and $70 \%$ of both sexes responded it was "because it elicits emotions and feelings", and in the second place came "to alleviate boredom”. Interestingly, the neural processes underlying aesthetic responses to music are much more clear and easily detectable scientifically than those elicited by the visual arts, probably because music has a more direct and powerful influence on subcortical emotional systems than the visual arts (Hirstein and Ramachandran, 1999).

Indeed, of great relevance to the use of music in clinical and therapeutic settings on the basis of its putative emotional powers is the notion that music elicits emotions rather than merely expresses an emotion that the listener recognizes. Actually, most people experience a particularly intense, euphoric response to music, frequently accompanied by an autonomic or psychophysiological component, described as “shivers-down-the-spine”, or “chills”. Indeed, listening to music automatically elicits physiological changes in blood circulation, respiration, skin conductivity, body temperature, heart rate, etc. (Krumhansl, 1997; Khalfa et al., 2002; Khalfa et al., 2008) which are autonomic responses of the sympathetic nervous system regulated by noradrenergic neurons in the brainstem and midbrain (Bernatzky et al., 2011; Chanda and Levitin, 2013). Recent research provides direct evidence of dopamine release in the striatal dopaminergic region during the experience of chills (Salimpoor et al, 2001)

Consistent with the autonomic responses of the sympathetic nervous system elicited by music, the available literature provides cogent evidence that like biologically relevant visual stimuli, music activates primitive structures in the limbic and paralimbic areas of the brain involved in reward and fear (Blood and Zatorre, 2001; Brown et al., 2004). Activation of limbic areas during listening to music was even observed in neonates (Perani et al., 2010). Therefore, music resonates with our basic emotional systems, bringing out many phylogenetically ancient affective emotions and it appears fair to assume that "our love of music reflects the ancestral ability of our mammalian brain to transmit and receive basic emotional sounds that can arouse affective feelings 
which are implicit indicators of evolutionary fitness” (Panksepp and Bernatzky, 2002, p. 134).

Yes, music elicits real emotions! However, in our view, crucial for the study of therapeutic uses of music emotions is understanding what the main structural components of music are, how these components elicit emotions and at what extent these components are universal and/or culturally determined. This knowledge is important for the understanding of neurophysiological data but also for the formulation of hypotheses and experimental designs grounded on knowledge about musical parameters and related emotional effects.

Evidence indicates that different configurations of musical characteristics can induce different emotions, and some of these characteristics are both universal and shared with language. Overall, literature indicates that emotions in music can vary across many dimensions, such as mode (major-like or minor-like scales), consonance/dissonance, pitch register, tempo (i.e. number of beats per minute, can be fast or slow), loudness, and complexity (Juslin and Sloboda, 2001; Laukka et al., 2013). Particularly tempo (fast or slow) and mode (major or minor), which are associated with listeners' arousal levels and moods, respectively, have been the most extensively examined central features underlying music emotions in comparison to other dimensions. Both major and minor scales are considered as mainly having consonant intervals, i.e. musical notes whose fundamental frequencies form small integer ratios with the first degree (Tonic), such as octave (2:1), perfect fifths (3:2) and fourths (4:3). The Western's major scale, however, contains more consonant intervals, such as a major third (5:4) and major sixth (5:3), compared to Western's minor scale which has a minor third (6:5) and minor sixth (8:3). Dissonant musical stimuli, in contrast, are those based on intervals whose fundamental frequencies stand in more complex ratios such as augmented fourth (45:32), minor second (16:15), and major seventh (15:8), this last being present in both major scale and harmonic minor scale commonly used in the Western music (Bidelman and Krishnan, 2009; Tramo et al, 2001).

Music is capable of inducing strong emotions with both positive and negative valence consistently across subjects (Krumhansl, 1997) and cultures (Laukka et al., 2013). Cross-culturally, individuals tend to readily associate melodies in major modes at fast tempos as happy and melodies in minor modes at slow tempos as sad, responses that are considered the most consistent emotional judgements in music (Fritz et al., 2009; Laukka et al., 2013). Whereas emotional responses to tempo are really precocious 
and appear to depend less on experience (Hannon and Trainor, 2007), judgements based solely on mode are evident only from 6 years of age (Dalla Bella et al., 2001).

Expectation (hence prediction) constitutes another basic component of music perception (Huron, 2006). It operates on a variety of levels, including melodic, harmonic, metrical, and rhythmic, and it addresses the question "what” and "when", that is, what tones or chords are expected to occur and when, in a given musical sequence. It is not only presumed to play an important role in how listeners group the sounded events into coherent patterns, but also to appreciate patterns of tension and relaxation contributing to music's emotional effects. Both cognitive and emotional responses to music depend on whether, when, and how the expectations are fulfilled (Juslin and Västfjäll, 2008). For instance, there is a hierarchy of stability in the tones forming the Western major scale in which the most stable note is the first degree (and the octave) of the scale, i.e. the Tonic, that gives to the listener a sensation of resolution. The Tonic is followed in stability by the fifth and the third scale tones, respectively, for their harmonic frequencies/components being more closely related to the Tonic; the stability decreases from fourth to sixth, with the second and seventh degrees the most unstable in this order. In the $\mathrm{C}$ major key this continuum from stability to instability will be $\mathrm{C}, \mathrm{G}$, E, F, A, D and B, respectively.

The main method used in cross-cultural comparisons of musical expectations is the probe tone task, first developed by Krumhansl and Shepard (1979) for quantifying the perceived hierarchy of stability of tones. Here a melody is presented to the listeners many times, but followed on each occasion by a single probe-tone with varying degree of fitness. Using a rating scale, the listeners assess the degree to which the probe-tone fits their expectations about how the melody might continue (Krumhansl et al., 2000). Cross-cultural studies comparing the fitness ratings given by Indian and Western listeners to North Indian ragas (Castellano et al., 1984), by Western and Balinese listeners to Balinese music (Kessler et al., 1984), native Chinese and American listeners’ responses to Chinese and British folk songs (see Thompson et al., 1997) all found strong agreement between listeners from these different musical cultures. Of course, there were effects of expertise depending on the listeners' familiarity with the particular musical culture.

Recent cross-cultural works on melodic expectancies, with Korean music (Nam, 1998) and with music of indigenous people of the Scandinavian Peninsula (Krumhansl et al., 2000), have provided additional evidence for the universal reliance on the 
hierarchical arrangement of pitches, indicating that music draws on common psychological principles of expectation even if musical cultures have a distinct effect in these principles, although the exact way it is accomplished varies with culture. Given the universal presence of consonant intervals in musical scales across cultures, it is reasonable to assume that consonance influences judgment in these probe tone tasks (Laukka et al., 2013). However, another factor underlying probe tone tasks after controlling for consonance/dissonance is the statistical properties of the music, such as the number of times that different tones and tone combinations appear in the presented musical contexts (Krumhansl and Cuddy, 2010).

Actually, an interaction between biology and culture appears to underlie the development of emotions. For instance, although evidence indicates that sad-happy judgments based on mode are the result of enculturation, being consistent around the age of six (DallaBella et al., 2001), this learning seems to depend on the sensitivity to consonance/dissonance (Cousineau, McDermott, and Peretz, 2012; Hannon and Trainor, 2007; Gosselin et al., 2015) which appears to be innate._Although findings on the innate preference for consonance in infants are mixed, sensitivity to consonance/dissonance and processing advantages for consonant stimuli are both present in listeners of all cultures, in young infants (Plantinga and Trehub, 2014; Virtala and Tervaniemi, 2017, but see also McDermott et al, 2016) and neonates as well (Perani et al., 2010). Indeed, just like adults, infants can detect minor interval changes in melodies of the same contour when melodies are based on consonant intervals, but not when based on dissonant intervals, and are also better in detecting subtle changes in consonant than in dissonant intervals (for a review see McDermott and Hauser, 2005). Perani and colleagues (2010) reported not only differential patterns of brain activations for consonant and dissonant musical stimuli in neonates, but also neural emotional responses in the limbic system for dissonance.

Evidence suggests that consonance/dissonance sensitivity appears to be the universal guide of scale construction across musical cultures of either extant (see Justus, and Hutsler, 2005) or extinct human societies (Conard et al., 2009).

Consonance/dissonance sensitivity, jointly with tempo and complexity (Laukka et al., 2013) is an important mechanism underlying valence-based judgments of music emotions cross-culturally (Fritz et al., 2009). A good evidence for the relevance of consonance/dissonance sensitivity to emotional evaluations based on mode comes from congenital amusics. Congenital amusia is a neurogenetic disorder apparently specific of 
musical abilities that affects, beyond singing in tune and dancing, pitch processing abilities such as melody discrimination and recognition, pitch direction, small pitch deviations, and sensitivity to consonance/dissonance (Cousineau et al., 2012; Cousineau et al., 2015; Gosselin et al., 2015). Congenital amusics’ deficits in fine-grained pitch perception, harmonicity perception, high abnormal perception of consonance and dissonance and no preference for consonance is causally associated with their inability to make happy-sad judgments based uniquely on mode changes (Cousineau et al., 2015; Gosselin, 2015).

We can conclude that substantial evidence suggests that sensitivity to consonance/dissonance is the main innate ability that serves as a fundamental building block of musical enculturation, such as the implicit knowledge of the hierarchical organization of the musical notes in the tonal system (notes and chords that best fit to complete the musical expectations and, hence, to conclude the song), and of the valence-based judgements of music (Hannon and Trainor, 2007; Laukka et al., 2013).

In short, musical excerpts played in major mode and fast tempos are frequently associated with happiness, whereas minor mode and slow tempos are considered sad. Considering tempo in isolation, fast-tempos is taken as happier than slow-tempos, although it is more correct to say that faster tempos reflect high-arousal emotions such as happiness, fear and anger, whereas slow tempos is used to express low-arousal emotions such as sadness, tenderness and love. In general, sounds that are loud, dissonant and fast induce high arousal with a negative valence of in the listeners and associated with negative and high arousal emotions such as anger, fear, whereas sounds that are smooth, consonant and at a slow or intermediate tempos induce low arousal and positive feelings such as peacefulness, love, etc., regardless of being music, speech or environmental sounds. Low-pitched sounds are less pleasant and associated with fear or anxiety and used to express negative valences in music. Unexpectedness and irregularity, jointly with dissonance, are consistently associated with negative valence of fear (Laukka et al., 2013; Vieillard et al., 2008).

\section{Music structure, music emotions and music therapy}

Whereas the mesostriatal pathway is involved in both motor and reward-related functions, the mesolimbic and mesocortical dopamine pathways, often referred as to mesocorticolimbic pathways, appear to be specifically dedicated to process rewarding stimuli (e.g. pleasure) and rewarding aspects of reinforcement learning (Wise, 2009). 
Mesocorticolimbic dopamine pathways are characterized by projections from the ventral tegmental area (mesencephalon) to nucleus accumbens (ventral striatum) and to the prefrontal cortex, respectively (Wise, 2009).

Imaging and lesion studies reveal the subcortical foundations of emotional musical experiences in many brain areas that are homologous between humans and all of the other mammals (Blood and Zatorre, 2001; Brown et al., 2004).

Pleasant/consonant music stimuli are systematically associated with activations of both paralimbic areas (e.g. insula, orbitofrontal cortex and ventromedial prefrontal cortex), involved in reward/motivation, emotion, and arousal, and the mesocorticolimbic dopamine pathways, which is the most important reward pathway; in contrast, unpleasant/dissonant music are associated with activations parahippocampal gyrus (Blood and Zatorre, 2001; Menon and Levitin, 2005), a paralimbic structure which, jointly with amygdalae, is involved in unpleasant emotional states evoked by pictures with negative emotional valence (Lane et al., 1997); the amygdalae, in its turn, is a key structure in fear processing, and has strong reciprocal connections with parahippocampal gyrus (Mesulam, 1998) which is deactivated with consonant music (Blood and Zatorre, 2001). Recently it has been demonstrated that unilateral damage to amygdala selectively impairs the perception of emotional expression of fear in scary music (minor chords on the third and sixth degrees, implying the use of many out-ofkey notes, and fast tempos), while recognition of happiness (major mode and fast tempo) was normal, and recognition of peacefulness (major mode and intermediate tempo played with pedal and arpeggio accompaniment) and sadness (minor mode at an average slow tempo) in music was less clearly affected by the medial temporal lobe resection (Gosselin et al., 2005; see also Khalfa et al., 2008). Further, patients with lesions in left amygdala show reduced hedonic pleasure in music listening (Griffiths et al., 2004).

We have seen that music can change heart rate variability (HRV), a measure of cardiac autonomic balance. For example, an early study (Uemura and Honda, 1998) showed an increases in HRV, an indicator of less stress and greater resilience, during listening to classical music, i.e. Coeur Fragile by Richard Clayderman (minor mode, slow tempo) and Waltz of the Flowers by Tchaikovsky (major mode and slow tempo), which also induced comfort in the listeners. In contrast, decreases in HRV, an indicator of greater stress, was observed during listening to rock music which induced discomfort. However, it is important to note that these results reflect the effects of the 
musical excerpts specifically selected for this study and not a necessary difference between classical music and rock. In fact, both classical musical excerpts were consonant and in slow tempo. Although the authors did not inform about the rock music, it is likely that it was in fast tempo and had a greater degree of dissonance.

Actually, relaxing music (consonant and in slow tempos) can reduce autonomic responses such as heart rate, blood pressure, and respiratory rate, whereas music excerpts eliciting high arousal emotions such as happy and fear, are characterized by faster tempos and accentuated rhythms, and are often associated with increases in respiration rate, heart rate and blood pressure and skin conductance as well which is better than other measures of the autonomic nervous system such as heart rate, because it is under strict control of the sympathetic branch of the nervous system (Khalfa et al., 2002). Reciprocally, sad music (slow tempo) has smaller responses on these autonomic measures when compared to happy music (Khalfa et al. 2002, 2008; Krumhansl, 1997). Some neurochemical findings are consistent with these results. In healthy subjects, stimulating music played at fast tempos, such as techno, increases plasma levels of stress hormones along the hypothalamic-pituitary-adrenal (HPA) axis , such as cortisol and adrenocorticotropic hormone, and other neurochemicals known to mediate stress response, such as norepinephrine (produced in the brainstem locus ceruleus and central and peripheral autonomic nervous system and known to regulate autonomic responses of heart rate, blood pressure, and respiration) and ß-endorphin. Consistently with the role of the amygdale in stress-related responses, this structure is "rich in cortisol receptors and interacts with norepinephrine input and hippocampal connections” (Chanda and Levitin, p. 183). In contrast, relaxing music (characterized by slow tempos and consonance) has been found to decrease these stress-related neurochemicals such as cortisol, norepinephrine and ß-endorphin (for a review see Chanda and Levitin, 2013). Interestingly, musical pleasure was shown to be associated with deactivation in the amygdala, supporting the anxiolytic effects of consonant music (Blood and Zatorre 2001).

It is important to remember that both relaxing and happy music are positively valenced and elicit activations of both mesostriatal and mesocortical dopamine pathways involved in reward. However, it is important to remind that dopamine is not the only neurochemical involved in reward (and could not be a 'pleasure' neurochemical per se), and the feeling of pleasure appears to depend on both the release of dopamine and endogenous opioids within the nucleus accumbens (Chanda and 
Levitin, 2013, p. 180). In fact, subjective feelings of pleasure is the product of dynamic interactions of neurochemical concentrations including dopamine, opiods and norepinephrin. Nevertheless, the subjective feelings of pleasure associated with consonant music (happy or calming) and its potential use to soothe pain appear to be related, among other things, to opioid transmission in the nucleus accumbens associated with dopamine release in the mesocorticolimbic pathways (Chanda and Levitin, 2013).

Despite several methodological limitations, an old study by Goldstein (1980) observed that by administering naloxone, a well-known opioid antagonist, responses of thrill and chills during music listening were attenuated, suggesting a causal link between positive-valenced music and release of endogenous opiods.

For its robust impact on emotions and socioemotional processes as well as for its associated psychophysiological effect, brain activations, and neurochemical effects (Bernatzky et al., 2011; Chanda and Levitin, 2013; Juslin and Sloboda, 2011; Koelsch, 2015), the potential of music as an effective medium for reducing anxiety, pain, stress and depression has been investigated.

In a meta-analysis including 51 studies using randomized controlled trials, Cepeda and colleagues (2006) concluded that adding music therapy to standard care in patients with chronic pain or cancer significantly reduced pain and opioid requirements. In a randomized clinical trial, Bringman et al. (2009) found that the relaxing music was more efficient than preoperative administration of the benzodiazepine midazolam. i.e. an anxiolytic drug used worldwide for sedation during minor operations and intensive care, with relaxing music.

In a randomized controlled clinical trial, Siedliecki and Good (2006) assigned their 60 subjects with chronic non-malignant pain syndromes (with back, neck and/or joint pain for at least 6 months and receiving at least one form of traditional medical or surgical pain management) to a music group with music selected by researchers, a music group with music selected by patients or a control group (without music intervention). Although no statistically significant difference was found between the two music groups, both groups had diminished pain and depression symptoms as well as better motor power and abilities in comparison to the control group.

In the same line, cancer patients have a high level of physical and psychological distress. Although therapeutic effects of music have not been clearly demonstrated in the end-of-life care (Bradt and Dileo, 2010), there is evidence that just listening to music can improve the psychological state of patients and promote their physical well- 
being in different oncological contexts (Richardson, Babiak-Vazquez, and Frenkel, 2008) including palliative care (Hilliard, 2005), radiotherapy (Dileo C, Bradt J, Grocke D, Magill, 2009) and chemotherapy (Lee et al., 2012). When combined with conventional cancer treatments, music therapy can alleviate anxiety and pain (Richardson et al., 2008) and also reduce analgesic requirements (Pyati and Gan, 2007).

The most distressing form of cancer treatment is chemotherapy during which patients are in high need of alleviating anxiety, pain and ameliorating psychological state as well. In a pilot study, Lee and colleagues (2012) carried out the first investigation that systematically compared the EEG responses to relaxation treatment using either monochord or progressive muscle relaxation, thus pioneering for providing information on the possible neural mechanisms underlying the therapeutic effects by music in the oncological context and for testing music therapy against a proven psychological relaxation method. Both groups of patients showed significant improvement in their physical and psychological states and in state anxiety. Further, the EEG signals for both groups showed an increase of posterior theta band $(3.5-7.5 \mathrm{~Hz}$ ) and a decrease of midfrontal beta-2 band (20-29.5 Hz) oscillations during the latter phase of music therapy session. Interestingly, these combination of EEG markers reflect brain's response to relaxed states. These results are also consistent with the findings by Sammler et al. (2007) who reported an increase of frontal midline theta power during listening to pleasant music. Furthermore, only the music therapy group showed a change in the neuronal complexity in the theta band oscillations (Bhattacharya and Lee, 2016).

Music-based interventions to reduce pain and anxiety, and promoting well-being also extend to children under different oncological (Barrera, Rykov, and Doyle, 2002; Daveson, 2001; Kain et al., 2004) and cardiac contexts (Hatem, Lira, and Mattos, 2006).

In general, the effectiveness of music as an additional medium to reduce pain in comparison to standard care has been demonstrated in a diversity of clinical populations since 1960's. However, most studies only compared treatments with and without music and did not inform about the action mechanisms underlying music-specific analgesic effects; a more parsimonious explanation could be that music just exerted a distraction effect. Roy and colleagues (2008) induced pain with thermal stimulations in the subjects to investigate the valence effects of pleasant (positive) and unpleasant (negative) musical excerpts which were matched in terms of arousal. Although valence did not change warmth perception and unpleasant music did not significantly affect pain, the 
pleasant excerpts, in contrast, significantly reduced pain intensity. This supports the notion that positive-valenced music contributes to analgesic effects.

One problem with the study by Roy and colleagues (2008) is that all pleasant musical stimuli were at fast tempos, i.e. high in arousal, to match with the high arousal levels of unpleasant excerpts. Therefore, further studies should be conducted to assess specific effects of high (fast tempos) and low (slow tempos) arousal in pleasant musical stimuli. Moreover, some of the pleasant excerpts in the study by Roy et al. (2008) were based on minor modes and some of those based on major modes had a certain degree of dissonance and modulations, thereby increasing the musical complexity. Music parameters like consonance/dissonance, mode and tempo, and complexity as well, are well known factors underlying music emotions.

Music, emotional communication and socio-cognitive therapy

Basic emotions (e.g. happiness, sadness, anger, and fear), are more easily communicated through music, and emotional prosody virtually share with music the same patterns in tempo, mode (major/minor), harmony, tonality, pitch, intonation, contour, interval, rhythm, amplitude, timbre, etc., that are specifically involved in communicating emotions (Juslin and Sloboda, 2011). Within the perspective that music and movement and music are inseparable we suggest that protomusical behaviors represent deeper links between music, language and social-cognition.

Human infants interact with their caregivers producing and responding to patterns of sound and action, a rhythmicity that is manifestation of a fundamental musical competence, a musicality that is part of a "natural drive in human sociocultural learning which begins in infancy" (Trevarthen, 1999, p. 194). Thus, innate sensitivity to pitch and rhythm structure does not seem to be in vain since infant-directed speech (also known as baby talk or motherese), a sing-song-like way adults instinctively use to communicate with children which is slower, with higher average pitch and exaggerated pitch contours which greatly facilitates speech perception and language acquisition (Kuhl, 2004; Trehub, 2003).

Beyond speaking melodiously, adults also sing play songs and lullabies to children, special genres of music whose common features among cultures are simple pitch contour, repetitions and narrow pitch range (Trehub, 2003). Musicality also allows infants to follow and respond accordingly to temporal regularities in vocalization, 
movement, and time, allowing the initiation of temporally regular sets of vocalizations and movements (Trevarthen, 1999).

These protomusical behaviors are so intertwined with protoverbal behaviors that "preverbal origins of musical skills cannot easily be differentiated from the prelinguistic stages of speech acquisition and from the basic alphabet of emotional communication” (Papousek, 1996b, p. 92). It is even argued that the musical elements that participate in the process of early communicative development "pave the way to linguistic capacities earlier than phonetic elements” (Papousek, 1996a, p. 43). In the pitch dimension, infantcaregiver interactions, cross-culturally, tend to exhibit the same range of characteristics such as exaggerated pitch contours on the caregiver's part ('motherese') and melodic modulation and primitive articulation on the infant's part, all in the context of the rhythmic and kinesthetic interactions. On the part of the infant, these activities develop into exploratory vocal play (between 4 and 6 months) which gives rise to repetitive babbling (from 7 to 11 months) from which emerges both variegated babbling and early words (between 9 and 13 months) (Kuhl, 2004; Papousek, 1996a, 1996b).

These temporally-controlled interactions involving synchrony and turn-taking are employed in the modulation and regulation of affective state (Dissanayake, 2000), and in the achievement and control of joint attention also referred as to 'primary intersubjectivity' (Trevarthen, 1999). Arguably, protomusical behaviors are often reciprocally imitative and also clearly emotionally charged and linked to social and emotion regulations in infancy. The turn-taking aspect of these games is the "rhythmic dance” between mother and child and adults across cultures play reciprocal imitative games with their children that embody the temporal turn-taking. As the infant develops, protomusic becomes music and continues to play this social role throughout life as an indispensable component of most diverse kinds of gatherings from occasional to magnificent ones, rituals and ceremonies (religious, social, healing, etc.). We know that imitation games with music and dance are universal, and the tribal dances itself can be seen as one of the most frequent forms of imitation game, used to develop the in-group sense, the feeling of both "being like the other" and "the other is like me", and thus pertaining to a group (Dissanayake, 2000; Trevarthenm 1999).

Finally, there is preliminary evidence that motherese (Seltzer, Ziegler, and Pollak, 2010) and relaxing music (Nilsson, 2009) are associated with increases in oxytocin a neuropeptide released by the posterior pituitary gland which is known to mediate social bonding and affiliation (for a review see Chanda and Levitin, 2013). 
It is in the universal role of music in the modulation and regulation of affective state and inter-subjectivity that relies the potential of music as an intervention tool in clinical contexts related to social communication impairments such as autism (Overy, and Molnar-Szakacs, 2009). Autism Spectrum Disorders (ASDs) are a group of neurological disorders characterized by social communication impairments, presence of stereotyped and repetitive behaviors and interests, with frequently co-ocurring motor impairments (Srinivasan, and Bhat, 2013). It was also recently demonstrated that mirror neurons in the posterior inferior frontal gyrus of high-functioning autistic children with autism showed no activity compared matched controls, during tasks involving imitation and observation of emotion expressions. Subserved by a fronto-parietal network in the human brain the mirror neuron system is fronto-parietal neural network involved in both other's action observation and execution thus allowing imitation processes and grasping of other's intention (theory of mind). The findings by Dapretto and colleagues (2006) indicate that social-cognitive deficits in autism could be due to a dysfunction of the mirror neuron system.

Since proto-musical behaviors are inextricably linked to imitation and emotionally charged and linked to social and emotion regulations in infancy, features that continue to characterize music behaviors throughout life, it is proposed that the human mirror neuron system and the limbic system interacts in the understanding of and attribution of emotion to complex musical patterns (Overy and Molnar-Szakacs 2006). In the Shared Affective Motion Experience model, SAME, proposed by Overy and Molnar-Szakacs (2009), music is not only patterned sound sequences but also the resulting intentionally and hierarchically organized sequences of expressive motor acts with emotional meaning, thus involving imitation, synchronization, emotion and social cognition. From this perspective, SAME model has important implications for music therapy and special education.

Improvisational music therapy, defined as the interactive use of live music for engaging clients to meet their therapeutic needs (Bruscia, 1998), resembles musical behaviors as typically occurring in the natural social contexts of proto-musical behaviors and shared and improvisational group activities (Juslin and Sloboda, 2011). Improvisational music therapy involves spontaneous self-expression, emotional communication and social engagement (Gold et al., 2006; Kim et al., 2009; Overy, and Molnar-Szakacs, 2009; Srinivasan, and Bhat, 2013). The use of improvisational music therapy with autistic children have been shown to enhance social skills by improving 
eye contact, social engagement, spontaneous initiation and emotional understanding, as well as verbal and gestural communication (Gold et al., 2006; Kim et al., 2009, see Srinavasan and Bhat, 2013).

\section{Music cognition and intervention in learning disabilities}

Music is a highly structured sequential organization of sounds and, like language, an acoustically based form of communication with a set of rules for combining limited number of perceptual discrete acoustic elements (pitches in music, and phonemes in language) in an infinite number of ways. According to the shared syntactic integration resource hypothesis (SSIRH) music and language can represent distinct modular systems at the level of long-term representations but share cognitive mechanisms underlying online structural integration of these representations (Patel, 2003) which appears to be inextricably linked to a domain general working memory system (Fedorenko et al. 2007).

Evidence for shared cognitive mechanisms and neural resources involved in tracking auditory patterned sequences and underlying intrinsic rules (syntax) between both domains has been demonstrated in behavioral (Fedorenko et al., 2009), imaging and lesion studies (see Koelsch, 2011). Similar evidence of likely sharing mechanisms has been made for children's linguistic abilities (phonology and literacy) and perception of musical sequences controlling for fine-grained pitch perception and rhythm (Zuk et al., 2013), as well as for the prosody of language and music (Patel et al., 2005; Zioga, Luft and Bhattacharya, 2016).

Music and language also seem to share some aspects of basic auditory perception. There is evidence that spectrotemporal auditory processing, particularly the processing of fast acoustic transitions, is essential for speech processing (and whose impairment can lead to reading disabilities) and that musical training can improve rapid temporal processing and reading as well (Gaab and Tallal, 2006).

It is also argued that accurate detection of supra-segmental cues, i.e. nonphonetic cues such as words, phrases and prosody (stress, rhythm and intonation), are key mechanisms underlying phonological development. Particularly the rhythmic prosody, such as perception of slow amplitude modulation in speech, is considered a key mechanism for segregating syllable onsets and rhymes which are essential for the acquisition of phonological representation in child development (Goswami et al., 2002). It is also proposed that infants build grammatical knowledge of the ambient language by 
means of this 'prosodic bootstrapping', with rhythm playing one of the central roles (Goswami et al., 2002; Corriveau et al., 2007).

Overlaps between music and other domains, such as auditory perception mechanism involved in speech perception and sequencing processing involved in syntax, is the first necessary requirement for hypothesizing the music can have an impact on these abilities. In fact, there is much evidence that musical experience shapes the brain and is associate with increases in white and gray matter in corpus callosum and auditory and motor cortices as well, and that musical training can have significant positive impact on academic abilities (Merret et al., 2013; Moreno et al., 2011; Schellenberg, 2004; Tierney and Kraus, 2013). These results can be taken as evidence for the great potential of music as an intervention tool for learning disabilities.

Nowadays literature abound with evidence the musical abilities correlate positively with language (e.g. phonological abilities) and literacy skills (Anvari et al., 2002; Zuk et al., 2013) and that musical training can improve speech perception abilities and neural coding of speech sounds (Schon et al., 2004; Kraus et al., 2009; Strait et al., 2012), phonological awareness and literacy abilities (Dege and Schwarzer, 2011; Register et al,, 2007), working-memory (Ribeiro and Santos, 2012) and executive functions (Moreno et al., 2011; Forgeard et al., 2008; Zuk et al., 2014), or even general intellectual abilities in the verbal and nonverbal domains (Schellenberg, 2004).

Overy (2003) reported both a correlation between song-rhythm tapping and spelling and dyslexic children's improvement in spelling after a music intervention based in song-rhythm tapping, suggesting that segmentation processes are common to both of these skills.

Consistent with the notion of sharing cognitive mechanisms in the perception of rhythm and processing of patterned auditory sequences musicianship has been shown to improve or correlate positively with language skills in numerous areas such as reading ability, phonological awareness, pitch processing in speech, prosody perception, and other language related abilities (for a brief review see Zuk et al., 2013).

Zuk et al. (2013) aimed at investigating the relations between music and language at the level of "patterned sequence processing" in a novel music task called Musical Sequence Transcription Task (MSTT). To control for fine-grained pitch processing they asked forty-three seven years old Brazilian students to listen to foursound sequences based on the combination of only two different sound types: one in the low register (thick sound), corresponding to a perfect fifth with fundamental frequencies 
$110 \mathrm{~Hz}(\mathrm{~A})$ and $165 \mathrm{~Hz}(\mathrm{E})$, and the other in the high register of (thin sound), corresponding to a perfect fourth, with $330 \mathrm{~Hz}$ (E) and $440 \mathrm{~Hz}$ (A). Children were required to mark the thin sound with a vertical line 'I' and the thick sound with an 'O', but were never told that the sequences only consist of four sounds. Performances of second graders on MSTT task were positively correlated with phonological processing and literacy skills, and predicted their literacy abilities 3 years after in the fifth grade (Figuccio, Andrade, Andrade, and Gaab, 2015). The authors claim that this task can potentially be used as a collective tool for the early screening for children at risk for reading disability.

For its characteristics and for improving social competence music is also a promising medium of intervention with populations handicapped in social skills in general, including the populations with Attention Deficit Hyperactivity Disorder (ADHD) (Rickson, 2006; Treurnich et al., 2011). However, while music has been shown to be somewhat effective for autistic children, the results in children with ADHD are mixed (Treurnich et al., 2011).

\section{Conclusion}

The field of neuroscience, especially the cognitive neuroscience, has been progressing rapidly. We now have myriad neuroimaging technologies available to reveal the intricate functioning of human brain operating across multiple spatiotemporal scales. This chapter presents an overview of the neuroscientific findings of music cognition and also attempts linking them with the working ingredients underlying music therapy. As an empirical research field, music therapy is in its infancy. Though cognitive neuroscience cannot answer sufficiently to all relevant issues in music therapy, we believe that with stronger dialogue between these two disciplines, our understanding about the underlying mechanisms of the healing power of music would be significantly improved (Magee and Stewart, 2015; O’Kelly, 2016).

\section{References}

Alvin J. Music therapy. Hutchinson; 1975.

Andrade PE, Bhattacharya J. Brain tuned to music. J R Soc Med. 2003 Jun;96(6):284-7. 
Andrade PE, Bhattacharya J. Music: Specialized to Integrate? Empirical Musicology Review, v. 9, n. 3-4, p. 183-192, 2015.

Andrade PE. Uma abordagem evolucionária e neurocientífica da música. Neurociencias. 2004, 1(1):21-33.

Anvari SH, Trainor LJ, Woodside J, Levy BA. Relations among musical skills, phonological processing, and early reading ability in preschool children. J Exp Child Psychol. 2002 Oct;83(2):111-30.

Barrera ME, Rykov MH, Doyle SL. The effects of interactive music therapy on hospitalized children with cancer: a pilot study. Psychooncology. 2002 SepOct;11(5):379-88.

Bernatzky G, Presch M, Anderson M, Panksepp J. Emotional foundations of music as a non-pharmacological pain management tool in modern medicine. Neurosci Biobehav Rev. 2011 Oct;35(9):1989-99. doi: 10.1016/j.neubiorev.2011.

Bhattacharya J, Lee EJ. Modulation of EEG theta band signal complexity by music therapy. Int J Bif Chaos. 2016 January; 1650001. doi: 10.1142/S0218127416500012.

Bidelman GM, Krishnan A. Neural correlates of consonance, dissonance, and the hierarchy of musical pitch in the human brainstem. J Neurosci. 2009 Oct 21;29(42):13165-71. doi: 10.1523/JNEUROSCI.3900-09.2009.

Blacking J. How Musical Is Man? Seattle: University of Washington Press; 1973.

Blood AJ, Zatorre RJ. Intensely pleasurable responses to music correlate with activity in brain regions implicated in reward and emotion. Proc Natl Acad Sci U S A. 2001 Sep 25;98(20):11818-23.

Bonny, H. L. (1986). Music and healing. Music Therapy, 6(1), 3-12.

Bradt J, Dileo C, Grocke D, Magill L. Music interventions for improving psychological and physical outcomes in cancer patients. Cochrane Database Syst Rev. 2011 Aug 10;(8):CD006911. doi: 10.1002/14651858.CD006911.pub2. Review.

Bradt J, Dileo C. Music therapy for end-of-life care. Cochrane Database Syst Rev. 2010 Jan 20;(1):CD007169. doi: 10.1002/14651858.CD007169.pub2. Review.

Bringman H, Giesecke K, Thörne A, Bringman S. Relaxing music as pre-medication before surgery: a randomised controlled trial. Acta Anaestesiol Scand 2009; 53: 759764 .

Brown S, Martinez MJ, Parsons LM. Passive music listening spontaneously engages limbic and paralimbic systems. Neuroreport. 2004 Sep 15;15(13):2033-7.

Bruscia KE. Standards of Integrity for Qualitative Music Therapy Research. J Music Ther. 1998;35(3):176-200. 
Bunt L, Stige, B. Music therapy: An art beyond words. Routledge; 2014.

Castellano MA, Bharucha JJ, Krumhansl CL. Tonal hierarchies in the music of north India. J Exp Psychol Gen. 1984 Sep;113(3):394-412.

Cepeda MS, Carr DB, Lau J, Alvarez H. Music for pain relief. Cochrane Database of Systematic Reviews 2006; 2:CD004843.

Chanda ML, Levitin DJ. The neurochemistry of music. Trends Cogn Sci. 2013 Apr;17(4):179-93. doi: 10.1016/j.tics.2013.02.007. Review.

Chen JL, Penhune VB, Zatorre RJ. Listening to musical rhythms recruits motor regions of the brain. Cereb Cortex. 2008 Dec;18(12):2844-54. doi: 10.1093/cercor/bhn042

Conard NJ, Malina M, Münzel SC. New flutes document the earliest musical tradition in southwestern Germany. Nature. 2009 Aug 6;460(7256):737-40. doi: 10.1038/nature08169. Epub 2009 Jun 24.

Corriveau K, Pasquini E, Goswami U. Basic auditory processing skills and specific language impairment: a new look at an old hypothesis. J Speech Lang Hear Res. 2007 Jun;50(3):647-66.

Cousineau M, McDermott JH, Peretz I. The basis of musical consonance as revealed by congenital amusia. Proc Natl Acad Sci U S A. 2012 Nov 27;109(48):19858-63. doi: 10.1073/pnas.1207989109.

Cousineau M, Oxenham AJ, Peretz I. Congenital amusia: a cognitive disorder limited to resolved harmonics and with no peripheral basis. Neuropsychologia. 2015 Jan;66:293301. doi: 10.1016/j.neuropsychologia.2014.11.031

Cross I. Music, cognition, culture, and evolution. Ann N Y Acad Sci 2001;930:28-42.

Dalla Bella S, Peretz I, Rousseau L, Gosselin N. A developmental study of the affective value of tempo and mode in music. Cognition. 2001 Jul;80(3):B1-10.

Dapretto M, Davies MS, Pfeifer JH, Scott AA, Sigman M, Bookheimer SY, Iacoboni M. Understanding emotions in others: mirror neuron dysfunction in children with autism spectrum disorders. Nat Neurosci. 2006 Jan;9(1):28-30.

Daveson, B. A. (2001). Music therapy and childhood cancer: Goals, methods, patient choice and control during diagnosis, intensive treatment, transplant and palliative care. Music Therapy Perspectives, 19(2), 114-120.

Degé F, Schwarzer G. The effect of a music program on phonological awareness in preschoolers. Front Psychol. 2011 Jun 20;2:124. doi: 10.3389/fpsyg.2011.00124.

Dissanayake, E. (2000). Antecedents of the temporal arts in early mother-infant interaction. In N. L. Wallin, B. Merker, \& S. Brown (Eds.), The origins of music (pp. 389-410). Cambridge, Mass: The MIT Press. 
Dissanayake, E. (2009). Root, leaf, blossom, or bole: Concerning the origin and adaptive function of music. Communicative musicality: Exploring the basis of human companionship, 17-30.

Fedorenko E, Gibson E, Rohde. The nature of working memory in linguistic, arithmetic and spatial integration processes. Journal of Memory and Language. 2007;56:246-269.

Fedorenko E, Patel A, Casasanto D, Winawer J, Gibson E. Structural integration in language and music: evidence for a shared system. Mem Cognit. 2009 Jan;37(1):1-9. doi: 10.3758/MC.37.1.1.

Fitch WT. The evolution of music in comparative perspective. Ann N Y Acad Sci. 2005 Dec;1060:29-49. Review.

Forgeard M, Winner E, Norton A, Schlaug G. Practicing a musical instrument in childhood is associated with enhanced verbal ability and nonverbal reasoning. PLoS One. 2008;3(10):e3566. doi: 10.1371/journal.pone.0003566

Fritz T, Jentschke S, Gosselin N, Sammler D, Peretz I, Turner R, Friederici AD, Koelsch S. Universal recognition of three basic emotions in music. Curr Biol. 2009 Apr 14;19(7):573-6. doi: 10.1016/j.cub.2009.02.058

Gold C, Wigram T, Elefant C. Music therapy for autistic spectrum disorder. Cochrane Database Syst Rev. 2006 Apr 19;(2):CD004381. Review.

Goldstein A. Thrills in response to music and other stimuli. Physiol. Psychol. 1980; 8, 126-129.

Gosselin N, Paquette S, Peretz I. Sensitivity to musical emotions in congenital amusia. Cortex. 2015 Oct;71:171-82. doi: 10.1016/j.cortex.2015.06.022

Gosselin N, Peretz I, Noulhiane M, Hasboun D, Beckett C, Baulac M, Samson S. Impaired recognition of scary music following unilateral temporal lobe excision. Brain. 2005 Mar;128(Pt 3):628-40.

Goswami U, Thomson J, Richardson U, Stainthorp R, Hughes D, Rosen S, Scott SK. Amplitude envelope onsets and developmental dyslexia: A new hypothesis. Proc Natl Acad Sci U S A. 2002 Aug 6;99(16):10911-6.

Gouk P (Ed.). Musical healing in cultural contexts. Ashgate Pub Limited; 2000

Griffiths TD, Warren JD, Dean JL, Howard D. "When the feelings gone”: a selective loss of musical emotion. J Neurol Neurosurg Psychiatr. 2004; 75: 344-345.

Halpern AR, Zatorre RJ. When that tune runs through your head: a PET investigation of auditory imagery for familiar melodies. Cereb Cortex. 1999 Oct-Nov;9(7):697-704.

Hannon EE, Trainor LJ. Music acquisition: effects of enculturation and formal training on development. Trends Cogn Sci. 2007 Nov;11(11):466-72. Epub 2007 Nov 5.

Review. 
Hatem TP, Lira PI, Mattos SS. The therapeutic effects of music in children following cardiac surgery. J Pediatr (Rio J). 2006 May-Jun;82(3):186-92.

Hillecke T, Nickel A, Bolay HV. Scientific perspectives on music therapy. Ann N Y Acad Sci. 2005 Dec;1060:271-82. Review.

Hilliard RE. Music Therapy in Hospice and Palliative Care: a Review of the Empirical Data. Evid Based Complement Alternat Med. 2005 Jun;2(2):173-178.

Honing H, ten Cate C, Peretz I, Trehub SE. Without it no music: cognition, biology and evolution of musicality. Philos Trans R Soc Lond B Biol Sci. 2015 Mar 19;370(1664):20140088. doi: 10.1098/rstb.2014.0088

Hughes AJ, Daniel SE, Kilford L, Lees AJ. Accuracy of clinical diagnosis of idiopathic Parkinson's disease: a clinico-pathological study of 100 cases. J Neurol Neurosurg Psychiatry. 1992 Mar;55(3):181-4.

Huron David. Sweet anticipation: Music and the psychology of expectation. MIT Press; 2006.

Hyde IH, Scalapino W. The influence of music upon electrocardiograms and blood pressure. American Journal of Physiology--Legacy Content, v. 46, n. 1, p. 35-38, 1918.

Janata P, Grafton ST. Swinging in the brain: shared neural substrates for behaviors related to sequencing and music. Nat Neurosci. 2003 Jul;6(7):682-7. Review.

Jankovic J. Parkinson's disease: clinical features and diagnosis. J Neurol Neurosurg Psychiatry. 2008 Apr;79(4):368-76. doi: 10.1136/jnnp.2007.131045. Review.

Juslin PN, Sloboda J (Ed.). Handbook of music and emotion: Theory, research, applications. OUP Oxford; 2011.

Juslin PN, Västfjäll D. Emotional responses to music: the need to consider underlying mechanisms. Behav Brain Sci. 2008; 31(5): 559-575.

Justus T, Hutsler JJ. Fundamental issues in the evolutionary psychology of music: Assessing innateness and domain specificity. Music Percept 2005; 23:1-27.

Kain ZN, Caldwell-Andrews AA, Krivutza DM, Weinberg ME, Gaal D, Wang SM, Mayes LC. Interactive music therapy as a treatment for preoperative anxiety in children: a randomized controlled trial. Anesth Analg. 2004 May;98(5):1260-6.

Kessler EJ, Hansen C, Shepard RN. Tonal schemata in the perception of music in Bali and in the West. Music Percept 1984;1:276-95.

Khalfa S, Isabelle P, Jean-Pierre B, Manon R. Event-related skin conductance responses to musical emotions in humans. Neurosci Lett. 2002 Aug 9;328(2):145-9. 
Khalfa S, Roy M, Rainville P, Dalla Bella S, Peretz I. Role of tempo entrainment in psychophysiological differentiation of happy and sad music? Int J Psychophysiol. 2008 Apr;68(1):17-26. doi: 10.1016/j.ijpsycho.2007.12.001

Kim J, Wigram T, Gold C. Emotional, motivational and interpersonal responsiveness of children with autism in improvisational music therapy. Autism. 2009 Jul;13(4):389-409. doi: $10.1177 / 1362361309105660$

Koelsch S. A neuroscientific perspective on music therapy. Ann N Y Acad Sci. 2009 Jul;1169:374-84. doi: 10.1111/j.1749-6632.2009.04592.x. Review.

Koelsch, S. Brain and music. West Sussex, UK: Wiley-Blackwell; 2012.

Koelsch S. Music-evoked emotions: principles, brain correlates, and implications for therapy. Ann N Y Acad Sci. 2015 Mar;1337:193-201. doi: 10.1111/nyas.12684

Koelsch S. Toward a neural basis of music perception - a review and updated model. Front Psychol. 2011 Jun 9;2:110. doi: 10.3389/fpsyg.2011.00110

Koelsch S. Towards a neural basis of music-evoked emotions. Trends Cogn Sci. 2010 Mar;14(3):131-7. doi: 10.1016/j.tics.2010.01.002.

Kraus N, Skoe E, Parbery-Clark A, Ashley R. Experience-induced malleability in neural encoding of pitch, timbre, and timing. Ann N Y Acad Sci. 2009 Jul;1169:543-57. doi: 10.1111/j.1749-6632.2009.04549.x. Review.

Krumhansl CL, Cuddy LL. A theory of tonal hierarchies in music. Music Percept 2010 36:51-87.

Krumhansl CL, Shepard RN. Quantification of the hierarchy of tonal functions within a diatonic context. J Exp Psychol Hum Percept Perform. 1979 Nov;5(4):579-94.

Krumhansl CL, Toivanen P, Eerola T, Toiviainen P, Järvinen T, Louhivuori J. Crosscultural music cognition: cognitive methodology applied to North Sami yoiks.

Cognition. 2000 Jul 14;76(1):13-58.

Krumhansl CL. An exploratory study of musical emotions and psychophysiology. Can J Exp Psychol. 1997 Dec;51(4):336-53.

Kuhl PK. Early language acquisition: cracking the speech code. Nat Rev Neurosci. 2004 Nov;5(11):831-43. Review.

Lane RD, Reiman EM, Bradley MM, Lang PJ, Ahern GL, Davidson RJ, Schwartz GE. Neuroanatomical correlates of pleasant and unpleasant emotion. Neuropsychologia. 1997 Nov;35(11):1437-44.

Laukka P, Eerola T, Thingujam NS, Yamasaki T, Beller G. Universal and culturespecific factors in the recognition and performance of musical affect expressions. Emotion. 2013 Jun;13(3):434-49. doi: 10.1037/a0031388. 
Lee EJ, Bhattacharya J, Sohn C, Verres R. Monochord sounds and progressive muscle relaxation reduce anxiety and improve relaxation during chemotherapy: a pilot EEG study. Complement Ther Med. 2012 Dec;20(6):409-16. doi:10.1016/j.ctim.2012.07.002

Levitin DJ, Tirovolas AK. Current advances in the cognitive neuroscience of music. Ann N Y Acad Sci. 2009 Mar;1156:211-31. doi: 10.1111/j.1749-6632.2009.04417.x.

Magee W, Stewart L. The challenges and benefits of a genuine partnership between music therapy and neuroscience: a dialog between scientist and therapist. Frontiers in Human Neuroscience. 2015 May; 9: 223.

McDermott JH, Hauser M. The origins of music: Innateness, uniqueness, and evolution. Music Percept. 2005;23:29-59.

McDermott JH. The evolution of music. Nature. 2008 May 15;453(7193):287-8. doi: 10.1038/453287a.

McDermott JH, Schultz AF, Undurraga EA, Godoy RA. Indifference to dissonance in native Amazonians reveals cultural variations in music perception. Nature. 2016 July 13; doi: 10.1038/nature18635.

Meister IG, Krings T, Foltys H, Boroojerdi B, Müller M, Töpper R, Thron A. Playing piano in the mind--an fMRI study on music imagery and performance in pianists. Brain Res Cogn Brain Res. 2004 May;19(3):219-28.

Menon V, Levitin DJ. The rewards of music listening: response and physiological connectivity of the mesolimbic system. Neuroimage. 2005 Oct 15;28(1):175-84.

Merker B, Morley I, Zuidema W. Five fundamental constraints on theories of the origins of music. Philos Trans R Soc Lond B Biol Sci. 2015 Mar 19;370(1664):20140095. doi: 10.1098/rstb.2014.0095. Review.

Merrett DL, Peretz I, Wilson SJ. Moderating variables of music training-induced neuroplasticity: a review and discussion. Front Psychol. 2013 Sep 9;4:606. doi: 10.3389/fpsyg.2013.00606. Review.

Merriam A. The anthropology of music. Evanston, Illinois: Northwestern University Press; 1964

Mesulam MM. From sensation to cognition. Brain. 1998 Jun;121 ( Pt 6):1013-52. Review

Mithen S. The singing Neanderthals: The origins of music, language, mind, and body. Cambridge, MA: Harvard University Press; 2006.

Moreno S, Bialystok E, Barac R, Schellenberg EG, Cepeda NJ, Chau T. Short-term music training enhances verbal intelligence and executive function. Psychol Sci. 2011 Nov;22(11):1425-33. doi: 10.1177/0956797611416999 
Nam, U. (1998). Pitch distribution in Korean court music. Evidence consistent with tonal hierarchies. Music Perception, 16 (2), 243-247.

Nilsson U. Soothing music can increase oxytocin levels during bed rest after open-heart surgery: a randomised control trial. J Clin Nurs. 2009 Aug;18(15):2153-61. doi: 10.1111/j.1365-2702.2008.02718.x

O’Kelly J. Music therapy and neuroscience: opportunities and challenges. Voices: A World Forum for Music Therapy. 2016; 16(2): 1-22.

Ohnishi T, Matsuda H, Asada T, Aruga M, Hirakata M, Nishikawa M, Katoh A, Imabayashi E. Functional anatomy of musical perception in musicians. Cereb Cortex. 2001 Aug;11(8):754-60.

Overy K, Molnar-Szakacs I. Being together in time: musical experience and the mirror neuron system. Music Percept 2009;26:489-504.

Panksepp J, Bernatzky G. Emotional sounds and the brain: the neuro-affective foundations of musical appreciation. Behav Processes. 2002 Nov;60(2):133-155.

Panksepp J. The emotional source of "chills" induced by music. Music Percept. 1995 13, 171-207.

Papousek, H. (1996a) Musicality in infancy research: biological and cultural origins of early musicality. In I. Deliège and J. Sloboda (eds) Musical Beginnings. Oxford: Oxford University Press, pp. 37-55.

Papousek, M. (1996b). Intuitive parenting: a hidden source of musical stimulation in infancy. In I. Deliège and J. Sloboda, (Eds.), Musical beginnings, Oxford: OUP

Patel AD, Foxton JM, Griffiths TD. Musically tone-deaf individuals have difficulty discriminating intonation contours extracted from speech. Brain Cogn. 2005 Dec;59(3):310-3.

Patel AD. Music, biological evolution, and the brain. Emerging disciplines. Bailar M, editor. Houston: Rice University Press; 2010.

Perani D, Saccuman MC, Scifo P, Spada D, Andreolli G, Rovelli R, Baldoli C, Koelsch S. Functional specializations for music processing in the human newborn brain. Proc Natl Acad Sci U S A. 2010 Mar 9;107(10):4758-63. doi: 10.1073/pnas.0909074107

Pinel JP. Biopsychology (8th ed.). Boston: Allyn \& Bacon; 2011.

Pinker S. How the Mind Works. New York: Norton; 1997.

Platel H, Price C, Baron JC, Wise R, Lambert J, Frackowiak RS, Lechevalier B, Eustache F. The structural components of music perception. A functional anatomical study. Brain. 1997 Feb;120 ( Pt 2):229-43. 
Pyati S, Gan TJ. Perioperative pain management. CNS Drugs. 2007;21(3):185-211. Review.

Ramachandran, V. S., \& Hirstein, W. (1999). The science of art: A neurological theory of aesthetic experience. Journal of consciousness Studies, 6(6-7), 15-51.

Register D, Darrow AA, Standley J, Swedberg O. The use of music to enhance reading skills of second grade students and students with reading disabilities. J Music Ther. 2007 Spring;44(1):23-37.

Repp BH, Su YH. Sensorimotor synchronization: a review of recent research (20062012). Psychon Bull Rev. 2013 Jun;20(3):403-52. doi: 10.3758/s13423-012-0371-2. Review.

Revisiting the innate preference for consonance. Plantinga J, Trehub SE. J Exp Psychol Hum Percept Perform. 2014 Feb;40(1):40-9. doi: 10.1037/a0033471.

Ribeiro, F. S., \& Santos, F. H. D. (2012). Musical training and working memory span in beginners, veterans and with no musical knowledge children. Psicologia: Reflexão e Crítica, 25(3), 559-567.

Richardson MM, Babiak-Vazquez AE, Frenkel MA. Music therapy in a comprehensive cancer center. J Soc Integr Oncol. 2008 Spring;6(2):76-81. Review.

Rickson DJ. Instructional and improvisational models of music therapy with adolescents who have attention deficit hyperactivity disorder (ADHD): a comparison of the effects on motor impulsivity. J Music Ther. 2006 Spring;43(1):39-62.

Roy M, Peretz I, Rainville P. Emotional valence contributes to music-induced analgesia. Pain. 2008 Jan;134(1-2):140-7.

Sakai K, Hikosaka O, Miyauchi S, Takino R, Tamada T, Iwata NK, Nielsen M. Neural representation of a rhythm depends on its interval ratio. J Neurosci. 1999 Nov 15;19(22):10074-81.

Salimpoor VN, Benovoy M, Larcher K, Dagher A, Zatorre RJ. Anatomically distinct dopamine release during anticipation and experience of peak emotion to music. Nature Neuroscience. 2011; 14: 257-262.

Sammler D, Grigutsch M, Fritz T, Koelsch S. Music and emotion: electrophysiological correlates of the processing of pleasant and unpleasant music. Psychophysiology. 2007 Mar;44(2):293-304.

Schellenberg EG. Music lessons enhance IQ. Psychol Sci. 2004 Aug;15(8):511-4.

Schön D, Magne C, Besson M. The music of speech: music training facilitates pitch processing in both music and language. Psychophysiology. 2004 May;41(3):341-9.

Seltzer LJ, Ziegler TE, Pollak SD. Social vocalizations can release oxytocin in humans. Proc Biol Sci. 2010 Sep 7;277(1694):2661-6. doi: 10.1098/rspb.2010.0567. 
Shannon, K. Neurologic music therapy: a scientific paradigm for clinical practice. Music and Medicine, v. 2, n. 2, p. 78-84, 2010.

Shepherd G. Neurobiology. Oxford: Oxford University Press; 1994.

Siedliecki SL, Good M. Effect of music on power, pain, depression and disability. J Adv Nurs. 2006 Jun;54(5):553-62.

Srinivasan SM, Bhat AN. A review of "music and movement" therapies for children with autism: embodied interventions for multisystem development. Front Integr Neurosci. 2013 Apr 9;7:22. doi: 10.3389/fnint.2013.00022

Strait DL, Parbery-Clark A, Hittner E, Kraus N. Musical training during early childhood enhances the neural encoding of speech in noise. Brain Lang. 2012 Dec;123(3):191201. doi: 10.1016/j.bandl.2012.09.001

Tallal P, Gaab N. Dynamic auditory processing, musical experience and language development. Trends Neurosci. 2006 Jul;29(7):382-90. Epub 2006 Jun 27. Review.

Teki S, Grube M, Kumar S, Griffiths TD. Distinct neural substrates of duration-based and beat-based auditory timing. J Neurosci. 2011 Mar 9;31(10):3805-12. doi: 10.1523/JNEUROSCI.5561-10.2011

Thaut MH, Abiru M. Rhythmic auditory stimulation in rehabilitation of movement disorders: a review of current research. Music Percept. 2010;27:263-269.

Thaut MH, McIntosh GC, Rice RR. Rhythmic facilitation of gait training in hemiparetic stroke rehabilitation. J Neurol Sci. 1997 Oct 22;151(2):207-12.

Thaut MH. Rhythm, Music, and the Brain: Scientific Foundations and Clinical Applications. New York: Taylor \& Francis; 2005.

Thompson WF, Cuddy LL, Plaus C. Expectancies generated by melodic intervals: evaluation of principles of melodic implication in a melody-completion task. Percept Psychophys. 1997 Oct;59(7):1069-76.

Tierney A, Kraus N. Music training for the development of reading skills. Prog Brain Res. 2013;207:209-41. doi: 10.1016/B978-0-444-63327-9.00008-4. Review.

Trainor LJ. The origins of music in auditory scene analysis and the roles of evolution and culture in musical creation. Philos Trans R Soc Lond B Biol Sci. 2015 Mar 19;370(1664):20140089. doi: 10.1098/rstb.2014.0089.

Tramo MJ, Cariani PA, Delgutte B, Braida LD. Neurobiological foundations for the theory of harmony in western tonal music. Ann N Y Acad Sci. 2001 Jun;930:92-116. Review.

Trehub SE. The developmental origins of musicality. Nat Neurosci. 2003 Jul;6(7):66973. Review. 
Treurnicht Naylor K, Kingsnorth S, Lamont A, McKeever P, Macarthur C. The effectiveness of music in pediatric healthcare: a systematic review of randomized controlled trials. Evid Based Complement Alternat Med. 2011;2011:464759. doi: $10.1155 / 2011 / 464759$

Trevarthen C. Autism as a neurodevelopmental disorder affecting communication and learning in early childhood: prenatal origins, post-natal course and effective educational support. Prostaglandins Leukot Essent Fatty Acids. 2000 Jul-Aug;63(1-2):41-6. Review.

Trevarthen, C. (1999). Musicality and the intrinsic motive pulse: evidence from human psychobiology and infant communication. Musicae Scientiae, Special Issue, 155-215.

Umemura M, Honda K. Influence of music on heart rate variability and comfort--a consideration through comparison of music and noise. J Hum Ergol (Tokyo). 1998 Dec;27(1-2):30-8.

Vieillard, S., Peretz, I., Gosselin, N., Khalfa, S., Gagnon, L., \& Bouchard, B. (2008). Happy, sad, scary and peaceful musical excerpts for research on emotions. Cognition \& Emotion, 22(4), 720-752.

Virtala, P, Tervaniemi, M. Neurocognition of major-minor and consonance-dissonance. Music Perception. 2017 April; 34(4), 387-404.

Wallin, NL, Merker B. The origins of music. MIT press; 2001.

Warth M, Kessler J, Koenig J, Wormit AF, Hillecke TK, Bardenheuer HJ. Music therapy to promote psychological and physiological relaxation in palliative care patients: protocol of a randomized controlled trial. BMC Palliat Care. 2014 Dec 17;13(1):60. doi: 10.1186/1472-684X-13-60.

Wheeler, B. L. (Eds). Music therapy handbook. New York: Guildford Press; 2016.

Wise RA. Roles for nigrostriatal--not just mesocorticolimbic--dopamine in reward and addiction. Trends Neurosci. 2009 Oct;32(10):517-24. doi: 10.1016/j.tins.2009.06.004

Zatorre R. Music, the food of neuroscience? Nature. 2005 Mar 17;434(7031):312-5.

Zatorre RJ, Chen JL, Penhune VB. When the brain plays music: auditory-motor interactions in music perception and production. Nat Rev Neurosci. 2007 Jul;8(7):54758. Review.

Zatorre RJ, Evans AC, Meyer E. Neural mechanisms underlying melodic perception and memory for pitch. J Neurosci. 1994 Apr;14(4):1908-19.

Zatorre RJ, Perry DW, Beckett CA, Westbury CF, Evans AC. Functional anatomy of musical processing in listeners with absolute pitch and relative pitch. Proc Natl Acad Sci U S A. 1998 Mar 17;95(6):3172-7. 
Zatorre RJ, Salimpoor VN. From perception to pleasure: music and its neural substrates. Proc Natl Acad Sci USA. 2013 Jun 18; 110: 10430-10437.

Zioga I, Luft CDB, Bhattacharya J. Musical training shapes neural responses to melodic and prosodic expectations. Brain Research. 2016 Nov 1650: 267-282.

Zuk J, Andrade PE, Andrade OV, Gardiner M, Gaab N. Musical, language, and reading abilities in early Portuguese readers. Front Psychol. 2013 Jun 18;4:288. doi: 10.3389/fpsyg.2013.00288

Zuk J, Benjamin C, Kenyon A, Gaab N. Behavioral and neural correlates of executive functioning in musicians and non-musicians. PLoS One. 2014 Jun 17;9(6):e99868. doi: 10.1371/journal.pone.0099868 